\title{
Intercâmbio internacional e sua perspectiva para enfermeiros e graduandos em Enfermagem: uma revisão integrativa
}

International exchange and its perspective for nurses and Nursing graduates: integrative review El intercambio internacional y su perspectiva para enfermeras y estudiantes de Enfermería: una revisión integradora

Fernanda Costa Martins Gallotti

ORCID: https://orcid.org/0000-0002-9063-1273 Universidade Federal de Sergipe, Brasil E-mail: fercosmart@gmail.com

Denisson dos Santos Andrade

ORCID: https://orcid.org/0000-0002-4259-3487 Universidade Tiradentes, Brasil

E-mail: dnssnandrade@gmail.com Letícia Freire Gonzaga

ORCID: https://orcid.org/0000-0001-6628-1210 Universidade Tiradentes, Brasil

E-mail: leticia.freire@souunit.com.br

Alice Tavares da Mota

ORCID: http://orcid.org/0000-0002-7364-921X Universidade Tiradentes, Brasil

E-mail: tavares.mota2012@hotmail.com

Luanna Feitosa

ORCID: https://orcid.org/0000-0003-0180-7538 Universidade Tiradentes, Brasil E-mail: lua.jem@gmail.com

Fernanda Dantas Barros

ORCID: https://orcid.org/0000-0001-9155-1665 Universidade Tiradentes, Brasil

E-mail: nanda-dantas@hotmail.com

Manuela de Carvalho Vieira Martins

ORCID: https://orcid.org/0000-0003-1222-5955

Universidade Tiradentes, Brasil

E-mail: manuela.cvm@hotmail.com

Shirley Dósea dos Santos Naziazeno

ORCID: https://orcid.org/0000-0002-9257-3150 Universidade Tiradentes, Brasil

E-mail: shirleydosea@yahoo.com.br

Maria Pureza Ramos de Santa Rosa

ORCID: https://orcid.org/0000-0003-4835-3043 Universidade Tiradentes, Brasil E-mail: maria_pureza@unit.br

Lenilson Santos da Trindade ORCID: https://orcid.org/0000-0002-4114-3740 Universidade Tiradentes, Brasil

E-mail: lenilsontrindade@msn.com

Mairim Russo Serafini

ORCID: https://orcid.org/0000-0003-4223-3470 Universidade Federal de Sergipe, Brasil

E-mail: maiserafini@hotmail.com

\section{Resumo}

Este estudo objetivou evidenciar as contribuições dos intercâmbios internacionais na formação e atuação do enfermeiro através do âmbito acadêmico, profissional e de pós-graduação. Trata-se de uma revisão integrativa realizada no período de julho a setembro de 2020 nas bases de dados PubMed, LILACS, Cochrane e Scielo. A seleção dos estudos por título e resumos e por leitura completa foi realizada por dois revisores de forma independente. Os estudos que geraram alguma discordância entre os revisores quanto à inclusão foram discutidos com um terceiro revisor. O processo de filtragem resultou em 49 artigos sobre intercâmbios internacionais, sendo desses, 75,5\% intercâmbio acadêmico, 16,3\% pós-graduação em enfermagem e $8,2 \%$ de capacitação profissional. Durante a análise dos referenciais teóricos envolvidos neste estudo, percebeu-se em todos os âmbitos de formação, tanto acadêmico como profissional, que a mobilidade internacional desenvolve no participante o saber técnico científico, agregando eficiência às práticas profissionais, além de um forte desenvolvimento pessoal. Conclui-se que a internacionalização em enfermagem é um diferencial potencial para o enfermeiro, seja este ainda em seu processo de formação, como graduando, seja como pós- 
graduando, ambos tendo a oportunidade de estudar e vivenciar outras metodologias de ensino, e até mesmo de conhecer e realizar práticas de enfermagem em um país distinto do de origem, melhorando suas competências profissionais.

Palavras-chave: Educação; Intercâmbios internacionais; Enfermagem.

\begin{abstract}
This study aimed to highlight the contributions of international exchanges in the training and performance of nurses through the academic, professional and postgraduate levels. This is an integrative review carried out from July to September 2020 in the PubMed, LILACS, Cochrane and Scielo databases. The selection of studies by title and abstracts and by complete reading was carried out by two reviewers independently. The studies that generated some disagreement among the reviewers regarding the inclusion were discussed with a third reviewer. The filtering process resulted in 49 articles on international exchanges, of which, $75.5 \%$ were academic exchanges, $16.3 \%$ were graduate students in nursing and $8.2 \%$ were professionally trained. During the analysis of the theoretical frameworks involved in this study, it was noticed in all areas of training, both academic and professional, that international mobility develops in the participant's scientific technical knowledge, adding efficiency to professional practices, in addition to strong personal development. It is concluded that internationalization in nursing is a potential differential for nurses, whether they are still in their training process, as a graduate student or as a postgraduate student, both having the opportunity to study and experience other teaching methodologies, and even to know and carry out nursing practices in a country other than the country of origin, improving their professional skills.
\end{abstract}

Keywords: Education; International exchanges; Nursing.

\title{
Resumen
}

Objetivo: Este estudio tuvo como objetivo resaltar los aportes de los intercambios internacionales en la formación y desempeño del enfermero a través de los niveles académico, profesional y de posgrado. Se trata de una revisión integradora realizada de julio a septiembre de 2020 en las bases de datos PubMed, LILACS, Cochrane y Scielo. La selección de estudios por título y resúmenes y por lectura completa fue realizada por dos revisores de forma independiente. Los estudios que generaron cierto desacuerdo entre los revisores con respecto a la inclusión se discutieron con un tercer revisor. El proceso de filtrado resultó en 49 artículos sobre intercambios internacionales, de los cuales, $75.5 \%$ fueron intercambios académicos, $16.3 \%$ fueron estudiantes de posgrado en enfermería y $8.2 \%$ tenían formación profesional. Durante el análisis de los marcos teóricos involucrados en este estudio, se advirtió en todas las áreas de la formación, tanto académica como profesional, que la movilidad internacional desarrolla en el participante conocimientos científico-técnicos, sumando eficiencia a las prácticas profesionales, además de un fuerte desarrollo personal. Se concluye que la internacionalización en enfermería es un potencial diferencial para el enfermero, ya sea que se encuentre aún en su proceso de formación, como estudiante de posgrado o como estudiante de posgrado, ambos teniendo la oportunidad de estudiar y experimentar otras metodologías de enseñanza, e incluso conocer y realizar prácticas de enfermería en un país distinto al de origen, mejorando sus competencias profesionales.

Palabras clave: Educación; Intercambios internacionales; Enfermería.

\section{Introdução}

O processo da globalização, definido como aumento do movimento de pessoas, ideias, capital, bens e serviços pelas fronteiras (Archanjo, 2017), está relacionado com a crescente tendência da mobilidade internacional acadêmica. Essa relação é perceptível principalmente no que se refere à disseminação de conhecimento por meio da maximização das relações interpessoais aproximando indivíduos, línguas e culturas (Guskuma, 2016).

Com o cenário da globalização, o movimento de internacionalização da educação superior ganhou destaque principalmente na década de 1990, pois, o "Acordo Geral sobre o Comércio de Serviços” (GATS), proposto pela Organização Mundial do Comércio (OMC), marcou o início do desenvolvimento da internacionalização do ensino no Brasil através do fornecimento de uma educação superior transfronteiriça, mobilidade internacional de estudantes e de professores (Franklin, Zuin \& Emmendoerfer, 2017).

A mobilidade acadêmica é considerada uma das principais estratégias para a formação dos estudantes e de aperfeiçoamento profissional. Entre 2013 e 2018, brasileiros foram co-autores de trabalhos de pesquisadores de 205 países, o que representou aproximadamente um terço de todos os trabalhos da Web of Science com autores brasileiros (Neves \& Barbosa, 2020). Com isso, é perceptível que além de adquirir conhecimento através do contato transcultural, o aluno 
intercambista pode contribuir no desenvolvimento social e tecnológico do país através de publicações de artigos científicos, agregando novos valores e competências ao futuro da profissão em ambos os países envolvidos.

Embora a mobilidade acadêmica não seja um fenômeno novo, a sua categoria de estudo tornou-se relevante a partir dos números de estudantes estrangeiros no país no início desse século. De acordo com a Organização das Nações Unidas para a Educação, a Ciência e a Cultura (UNESCO), o número de estudantes internacionais em todo o mundo aumentou de cerca de 2 milhões para 5 milhões entre 2000 e 2017. Em 2010, o Brasil recebeu apenas 37\% de estudantes em relação aos enviados para o exterior, porém em 2014 esse cenário mudou passando para 48,44\%, pois foram enviados 40.981 brasileiros para o exterior e 19.855 estudantes estrangeiros vieram estudar no Brasil (Pinto \& Larrechea, 2018).

A internacionalização dos programas de Pós-Graduação e estágios de aperfeiçoamento profissional também são fatores em crescimento no país. De acordo com o GEOCAPES - Sistema de Informações Georreferenciadas da Coordenação de Aperfeiçoamento de Pessoal Nível Superior, o número de distribuição de bolsas de pós-graduação, incluindo capacitação profissional no exterior, ultrapassa um total de 5.400 e no âmbito da enfermagem, entre 2018-2019, esse número chegou a 131 bolsas para o exterior em diversos países (Geocapes, 2020).

Nesse contexto, o processo de educação em enfermagem ao basear-se num currículo por competências, está em constante transformação visando uma tentativa de preparar, motivar e empoderar os enfermeiros para assumirem o protagonismo junto às políticas de saúde, com um nível de liderança transformacional, num contexto interprofissional (Ximenes Neto et al., 2020).

Portanto, ter a oportunidade de estudar em um país distinto do de origem permite ao graduando ou profissional de enfermagem conhecer o sistema de sua profissão em uma diferente realidade, podendo assim comparar e observar as diferenças e semelhanças existentes nos país de origem e destino (Jung et al., 2015). Dessa forma, será possível realizar uma auto análise de como está sendo seu processo de cuidar, identificando o que pode ser mudado ou agregado, como exemplo, a consciência cultural, empatia, adaptabilidade entre culturas, habilidades de comunicação e competência intercultural (Holtbrugge \& Engelhard, 2016) que por certo irão culminar na prestação de uma assistência de excelência ao paciente, trazendo um diferencial à sua realidade profissional.

Diante do exposto, fica evidente o número crescente de intercâmbio internacional nas últimas décadas e a necessidade de explorar as contribuições acerca da internacionalização e sua repercussão para o cenário da enfermagem. $\mathrm{O}$ presente trabalho, por sua vez, optou por discutir, por meio de uma revisão integrativa, a repercussão do intercâmbio internacional em enfermagem em três modalidades: acadêmico, profissional e de pós-graduação, tendo por objetivo evidenciar as contribuições dos intercâmbios internacionais na formação e atuação do enfermeiro através do âmbito acadêmico, profissional e de pós-graduação.

\section{Metodologia}

Este estudo compreende uma revisão integrativa realizada no período de julho a setembro de 2020, com propósito de responder às seguintes questões: De qual forma o intercâmbio internacional contribui na formação e atuação do profissional enfermeiro?

\subsection{Critérios de elegibilidade e estratégia de busca}

Em pesquisa preliminar nos bancos de dados Cochrane Database of Systematic Reviews, a Joanna Briggs Institute Library of Systematic Reviews e o PubMed outras pesquisas de revisão relacionada à intercâmbio internacional na formação do enfermeiro foram encontradas, no entanto, com o delineamento de objetivos diferentes do proposto nesta pesquisa. 
Foi realizada uma busca preliminar no portal PubMed para identificar termos controlados e não controlados contidos em títulos e resumos de estudos. As pesquisas foram realizadas por dois revisores de forma independente, os termos de pesquisa e estratégias desenvolvidas pelo revisor principal foram validados por pares.

As bases de dados utilizadas foram: PubMed, LILACS, Cochrane e Scielo. Nas bases PUBMED e Cochrane foi aplicada a estratégia de busca com descritores MeSH (Medical Subject Headings) "International Educational Exchange" [MeSH], "Education, nursing" [MeSH]. Já nas bases de dados LILACS e Scielo foram utilizados DECs (Descritores em Ciências da Saúde) "Intercâmbio Educacional Internacional", "Educação em Enfermagem", associados ao operador booleano and para as combinações entre os termos. A seleção dos estudos por título e resumos e por leitura completa foi realizada pelo revisor primário (D.S.A.) e secundário (L.F.G.) de forma independente. Os estudos que geraram alguma discordância entre os revisores quanto à inclusão foram discutidos com um terceiro revisor (F.C.M.G.).

Os critérios de inclusão aplicados nesta pesquisa foram: estudos publicados na íntegra em português, espanhol ou inglês, que apresentassem como objeto de investigação as implicações do intercâmbio internacional na formação e atuação do profissional enfermeiro. Foram excluídos os estudos que não apresentaram o texto completo online ou em coleção bibliográfica ou após contato direto com os autores do manuscrito. Com isso, não foi delimitado corte temporal para a busca, sendo considerado todos os artigos até agosto de 2020 publicados nas bases de dados selecionadas.

\subsection{Análise dos dados}

Os resultados foram apresentados por meio de análise descritiva e exploratória dos dados, evidenciando a relação / contribuições entre os intercâmbios internacionais e a formação e a atuação dos enfermeiros.

\section{Resultados}

Foram selecionados 269 artigos para leitura na íntegra, sendo excluídos nessa etapa 220 artigos proveniente dos motivos: artigos que tangenciam o tema proposto, não disponíveis para leitura na íntegra, abordam metodologias para estabelecer programas privados de intercâmbio internacional acadêmico em universidades, fatores que motivam alunos a realizarem o intercâmbio, questões de colaboração entre instituições e intercâmbios de forma virtualizada e sistema de comunicação por correspondência com estrangeiros (Figura 1). 
Figura 1 - Fluxograma dos estudos incluídos.

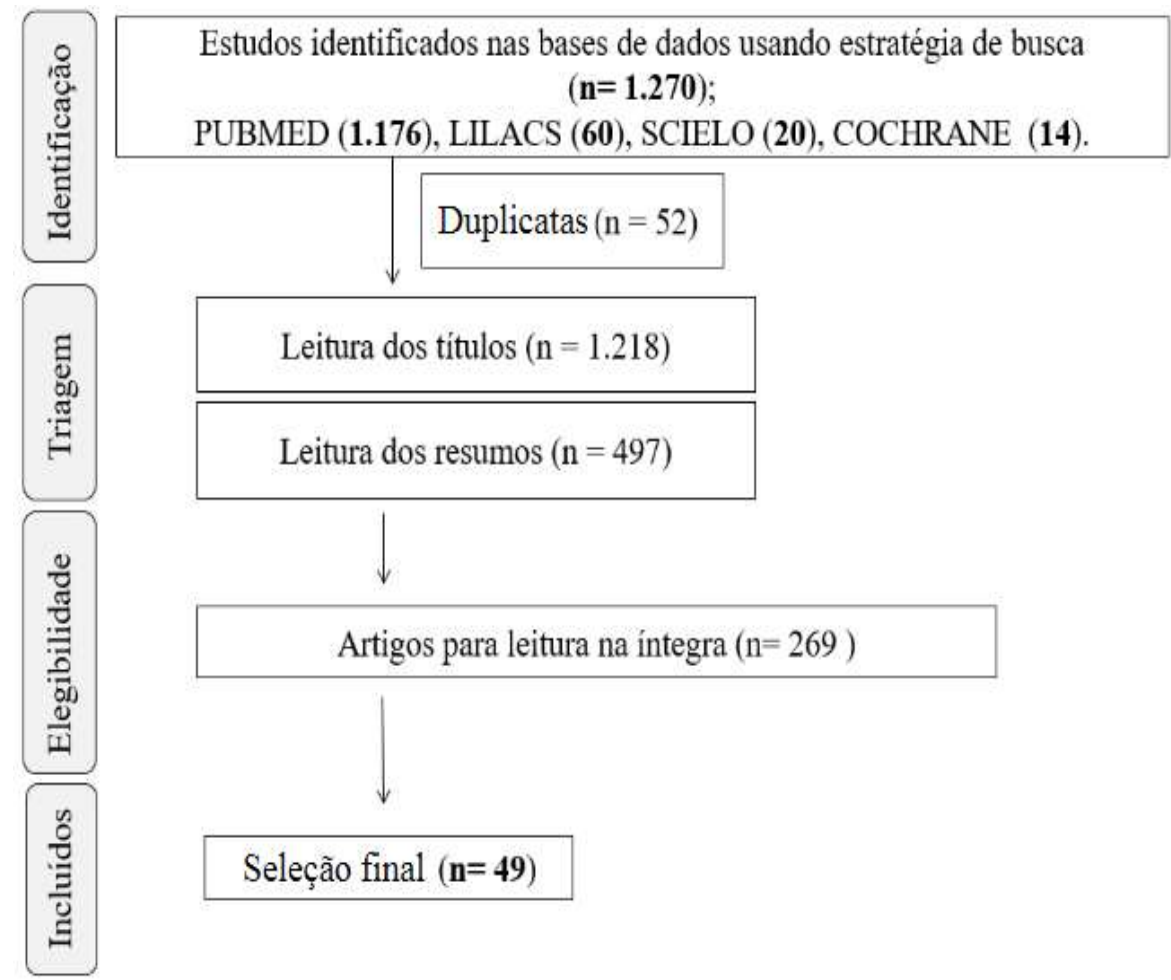

Fonte: Autores (2020).

O processo de seleção resultou em 49 artigos sobre intercâmbios internacionais, sendo desses, 75,5\% intercâmbio acadêmico, $16,3 \%$ pós-graduação em enfermagem e $8,2 \%$ de capacitação profissional.

Figura 2 - Distribuição em gráfico de linhas com o número de artigos selecionados e ano de publicação.

Número de artigos segundo ano de publicação

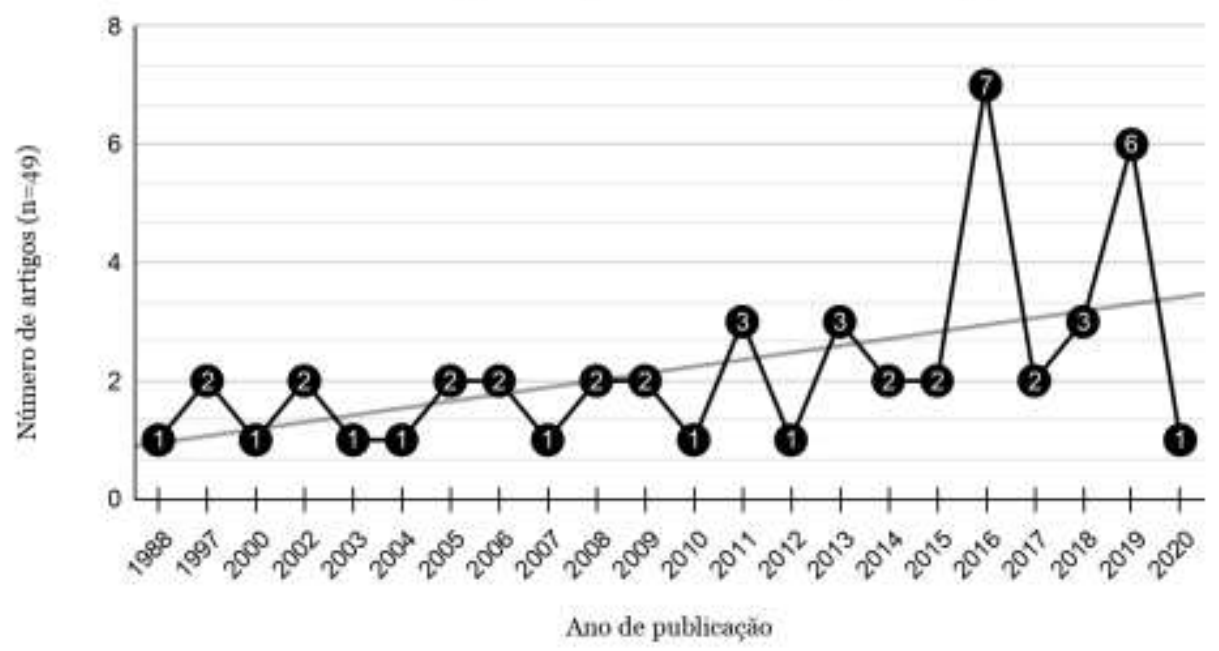

Fonte: Autores (2020). 
A partir da análise da Figura 2 percebe-se que o maior número de publicações foi observado no ano de 2016 seguido do ano de 2019, com sete e seis artigos publicados respectivamente. Em contrapartida, os anos 1988, 2000, 2003, 2004, 2007, 2010, 2012 e 2020 obtiveram o menor número de artigos publicados. Em complementação, o gráfico apresenta uma linha de tendência crescente em relação ao ano e número de publicações, que sugerem um aumento dos intercâmbios internacionais, consequentemente de estudos, como mencionado anteriormente (UNESCO, 2018).

Os resultados contemplam as três modalidades de intercâmbio internacional para esse estudo, sendo representados por intercâmbio acadêmico (Quadro 1), pós-graduação (Quadro 2) e profissional (Quadro 3), com o propósito de organizar e sintetizar as informações, contemplando os seguintes ítens: autores, ano de publicação e principais resultados.

Inicialmente apresenta-se um quadro composto por 37 artigos que descrevem sobre mobilidade internacional realizada por graduandos em enfermagem elencados no Quadro 1 a seguir.

Quadro 1 - Apresentação dos artigos incluídos para o estudo no âmbito de intercâmbio acadêmico internacional (n= 37).

\begin{tabular}{|c|c|}
\hline \multicolumn{2}{|r|}{ INTERCÂMBIO ACADÊMICO INTERNACIONAL } \\
\hline Autor/Ano & Principais Resultados \\
\hline ANDERSON, 2013 & $\begin{array}{l}\text { Independência, autonomia e senso de confiança sobre sua capacidade de cumprir } \\
\text { metas e resultados; }\end{array}$ \\
\hline BUBADUÉ, 2013. & $\begin{array}{l}\text { Protagonismo e autonomia no processo do planejamento do cuidado, o crescimento } \\
\text { acadêmico, pessoal e social }\end{array}$ \\
\hline CALDWELL, PURTZER, 2015. & $\begin{array}{l}\text { Aprimoramento das competências linguísticas, enfrentamento a solução de } \\
\text { problemas, desenvolvimento de habilidades técnicas e científicas. }\end{array}$ \\
\hline CALLISTER, COX, 2006. & $\begin{array}{l}\text { Enfermeiros educadores foram encarregados de aumentar a ênfase em seus } \\
\text { programas em competência cultural com base em resultados de pesquisas atuais. }\end{array}$ \\
\hline $\begin{array}{l}\text { CARVALHO,BACKES, LOMBA, } \\
\text { COLOMÉ,2016. } \\
\end{array}$ & $\begin{array}{l}\text { A análise dos dados resultou na possibilidade de desenvolvimento da autonomia dos } \\
\text { estudantes e contribuições culturais no processo do intercâmbio académico. }\end{array}$ \\
\hline COUTO, PEREIRA, 2018. & $\begin{array}{l}\text { Contribuiu na construção do indivíduo como profissional, através da estimulação do } \\
\text { pensamento crítico, após o contato com um sistema de saúde diferente. }\end{array}$ \\
\hline DEDEE, STEWART, 2003. & $\begin{array}{l}\text { Reflexões sobre os impactos a longo prazo na vida profissional após a experiência } \\
\text { internacional. }\end{array}$ \\
\hline $\begin{array}{l}\text { DUFFY, FARMER, RAVERT, } \\
\text { HUITTINEN, } 2005 .\end{array}$ & $\begin{array}{l}\text { Os graduados pontuaram o desenvolvimento e sensibilidade cultural após a } \\
\text { experiência. }\end{array}$ \\
\hline $\begin{array}{l}\text { DYCHES, HAYNES-FERERE, } \\
\text { HAYNES, } 2019 .\end{array}$ & Melhor compreensão cultural e autopercepção. \\
\hline FENNELL, 2009. & $\begin{array}{l}\text { O programa teve um impacto profundo nesses alunos que reforçam a importância da } \\
\text { saúde internacional e do estudo no exterior para estudantes universitários. }\end{array}$ \\
\hline FERRILLO, 2020. & $\begin{array}{l}\text { Houve diferenças ao comparar grupos que tiveram experiência internacional e os que } \\
\text { não tiveram }\end{array}$ \\
\hline GIRONDI,2016 & $\begin{array}{l}\text { Aperfeiçoamento da formação acadêmica, cultural e pessoal, a partir da inter- } \\
\text { relação entre teoria e prática internacional. }\end{array}$ \\
\hline GOSSE, KATIC-DUFFY, 2019. & $\begin{array}{l}\text { Ganhos na aquisição de conhecimento, melhoria da competência cultural, bem como } \\
\text { transformação pessoal e profissional. }\end{array}$ \\
\hline GREEN, 2008. & $\begin{array}{l}\text { Desenvolvimento profissional de conhecimento e habilidades práticas e um aumento } \\
\text { de consciência das diferenças nas práticas de saúde. }\end{array}$ \\
\hline $\begin{array}{l}\text { GUEDES, CAVALCANTE, } \\
\text { PUSCHEL, } 2017 .\end{array}$ & $\begin{array}{l}\text { A vivência do intercâmbio durante a graduação permitiu o desenvolvimento da sua } \\
\text { autonomia, autoconfiança e controle emocional. }\end{array}$ \\
\hline GUSKUMA, 2016. & $\begin{array}{l}\text { Temas emergiram revelando evidências de aprendizagem de longo prazo como } \\
\text { desenvolvimento cultural e pessoal. }\end{array}$ \\
\hline
\end{tabular}


Quadro 1 - Apresentação dos artigos incluídos para o estudo no âmbito de intercâmbio acadêmico internacional (n=37) (Continuação). Fonte: Autores (2020).

\begin{tabular}{|c|c|}
\hline Autor/Ano & Principais Resultados \\
\hline $\begin{array}{l}\text { KADDOURA,PURI,DOMI } \\
\text { NICK,2013. }\end{array}$ & $\begin{array}{l}\text { Valorização entre profissionais de saúde, cultura e diferenças, aproveitando a experiência e o } \\
\text { pensamento crítico na sua prática. }\end{array}$ \\
\hline KOHLBRY, 2016. & Estimulação da competência cultural entre diversos participantes do estudo. \\
\hline $\begin{array}{l}\text { KOSKINEN, JOKINEN, } \\
2007 .\end{array}$ & $\begin{array}{l}\text { A competência intercultural foi definida como um processo de aprendizagem, } \\
\text { autoconsciência e amadurecimento pessoal; procede através de cognitivo, emocional e } \\
\text { comportamental. }\end{array}$ \\
\hline LAW , MUIR, 2006. & $\begin{array}{l}\text { Maior eficácia da cooperação internacional; maior respeito pela identidade e diversidade/ } \\
\text { interação pessoal intensificada; melhores relações de trabalho; }\end{array}$ \\
\hline MAAS, 2011. & $\begin{array}{l}\text { As percepções dentro de outro sistema de saúde podem contribuir para melhorar o } \\
\text { atendimento ao paciente. }\end{array}$ \\
\hline $\begin{array}{l}\text { MILES, ERAMAA, } \\
\text { PARKKINEN, } 2019 .\end{array}$ & $\begin{array}{l}\text { Trabalhar com estrangeiros aumentou as compreensões dos alunos das questões de saúde em } \\
\text { outra cultura. }\end{array}$ \\
\hline MORGAN, 2019. & $\begin{array}{l}\text {.Os alunos tornaram-se diferentes em vários aspectos de como eram antes da experiência } \\
\text { internacional }\end{array}$ \\
\hline MWENDA, 2012. & $\begin{array}{l}\text { A exposição dos alunos a novos ambientes culturais, diferentes organizações de sistemas de } \\
\text { saúde influenciam a vida acadêmica e pessoal além de tornar a educação global. }\end{array}$ \\
\hline MYHRE, 2011. & Maior autoconfiança e compreensão dos conceitos fundamentais em enfermagem. \\
\hline NJ LEE, 2004. & $\begin{array}{l}\text { Os alunos identificaram como as experiências poderiam influenciar em situações futuras na } \\
\text { prática de enfermagem. }\end{array}$ \\
\hline $\begin{array}{l}\text { ORTEGA,MITCHELL, } \\
\text { PERAGALLO, } 2016 .\end{array}$ & $\begin{array}{l}\text { O aprendizado sobre diferentes culturas, sistemas de saúde e funções profissionais têm um } \\
\text { impacto significativo em suas práticas futuras. }\end{array}$ \\
\hline $\begin{array}{l}\text { PENINNAH, KLINGBEIL, } \\
2019 .\end{array}$ & $\begin{array}{l}\text { A experiência foi uma ferramenta valiosa para tornar os alunos culturalmente humildes e } \\
\text { fornecer cuidados de saúde culturalmente seguros. }\end{array}$ \\
\hline $\begin{array}{l}\text { ROLLS, INGLIS, KRISTY, } \\
1997 .\end{array}$ & $\begin{array}{l}\text { Desenvolvimento pessoal aumentado, autoconfiança e consciência dos alunos sobre cuidados } \\
\text { e necessidades de saúde. }\end{array}$ \\
\hline $\begin{array}{l}\text { RYANRENEE S. } \\
\text { TWIBELL, } 2002 .\end{array}$ & Crescimento pessoal e profissional; resultado positivo da mudança na prática de enfermagem. \\
\hline SMITH, CURRY, 2011 & $\begin{array}{l}\text { Destacou-se os itens de dimensão profissional, pessoal e intelectual desenvolvidas durante a } \\
\text { experiência internacional }\end{array}$ \\
\hline SMITH-MILLER, 2010. & Aumento na desenvoltura, pensamento crítico e habilidades de resolução de problemas. \\
\hline STEEN, ZDECHLIK, 2016. & $\begin{array}{l}\text { Aumentou o nível de conforto ao trabalhar com pacientes de culturas diferentes bem como o } \\
\text { crescimento cultural e pessoal. }\end{array}$ \\
\hline
\end{tabular}


Quadro 1 - Apresentação dos artigos incluídos para o estudo no âmbito de intercâmbio acadêmico internacional (n= 37) (conclusão).

\begin{tabular}{|c|l|}
\hline $\begin{array}{c}\text { Autor/Ano } \\
\text { STONE, } \\
\text { RIET,JUNLAPEEY } \\
\text { A, 2014. }\end{array}$ & $\begin{array}{c}\text { Pesenvolvimento de etno-empatia cultural, com potencial para transformar sua futura prática como } \\
\text { profissional de saúde, incorporando o novo aprendizado. }\end{array}$ \\
\hline $\begin{array}{c}\text { THOMPSON, } \\
\text { BOORE, DEENY, } \\
2000 .\end{array}$ & $\begin{array}{l}\text { Desenvolvimento pessoal e profissional, além de melhorar a compreensão dos alunos sobre a } \\
\text { influência de fatores culturais sobre saúde e cuidados de saúde. }\end{array}$ \\
\hline $\begin{array}{c}\text { ULVUND, } \\
\text { MORDAL, 2017. }\end{array}$ & Forçou um processo contínuo pelo qual os alunos desenvolveram a competência cultural. \\
\hline WESTERBOTN, & $\begin{array}{l}\text { Tanto o desenvolvimento pessoal quanto o profissional foram expressados pelos alunos; aumento da } \\
\text { autoconfiança e autoconsciência. }\end{array}$ \\
\hline
\end{tabular}

Fonte: Autores (2020).

No Quadro 2 logo abaixo, foram elencados oito artigos que descrevem sobre intercâmbio internacional para pósgraduandos, onde são descritos os resultados da experiência realizada pelos mesmos em suas pesquisas científicas internacionais.

Quadro 2 - Apresentação dos artigos incluídos no âmbito da pós-graduação internacional em enfermagem (n=8).

\begin{tabular}{|c|c|}
\hline \multicolumn{2}{|r|}{ PÓS-GRADUAÇÃO INTERNACIONAL } \\
\hline Autor/Local & Principais Resultados \\
\hline ANDREWS, 1988. & $\begin{array}{l}\text { Os programas de educação em enfermagem não fornecem enfermeiras com a preparação acadêmica } \\
\text { necessária para funcionar eficazmente na arena internacional. }\end{array}$ \\
\hline $\begin{array}{l}\text { BARDAQUIM, } \\
\text { DIAS, } 2019 .\end{array}$ & $\begin{array}{l}\text { Autonomia para resolução de problemas, desenvolvimento intelectual e profissional através de } \\
\text { palestras, eventos e discussões de trabalhos científicos. }\end{array}$ \\
\hline CARTY, 2002. & Os problemas incluem a prontidão para papéis de liderança internacional, preparados com doutorado. \\
\hline $\begin{array}{l}\text { FIGUEIREDO, } \\
\text { FERNANDES, } \\
\text { AYALA, } 2018 .\end{array}$ & $\begin{array}{l}\text { Formação de vínculos acadêmicos, contribuição para formação de habilidades interpessoais e } \\
\text { profissionais permeadas pela humanização da assistência e para o processo de internacionalização do } \\
\text { curso de enfermagem. }\end{array}$ \\
\hline HINSHAW, 1997. & $\begin{array}{l}\text { A troca de acadêmicos de doutorado e pós-doutorado amplia os horizontes de oportunidades e } \\
\text { compreensão em todos os países, melhorando cada programa de doutorado especificamente. }\end{array}$ \\
\hline
\end{tabular}


Quadro 2 - Apresentação dos artigos incluídos no âmbito da pós-graduação internacional em enfermagem (n=8) (Conclusão).

\begin{tabular}{|c|l|}
\hline Autor/Ano & \multicolumn{1}{c|}{ Principais Resultados } \\
\hline LORENZINI, 2016. & $\begin{array}{l}\text { Contribuição científica, desenvolvimento de outros idiomas, além do contato com outros } \\
\text { estudantes favorecendo a estimulação de pesquisa. }\end{array}$ \\
\hline MAAS, 2014. & $\begin{array}{l}\text { Promoveu uma compreensão global do desenvolvimento acadêmico e profissional exclusivo do } \\
\text { aluno de enfermagem de prática avançada. }\end{array}$ \\
\hline NORA, 2018. & Permitiu a participação ativa em eventos de pesquisa estimulando seu desempenho intelectual. \\
\hline
\end{tabular}

Fonte: Autores (2020).

Por último, quatro artigos mostram os resultados de profissionais enfermeiros que decidiram sair de seus países para viver a prática profissional em um país distinto, por um determinado tempo, demonstrados no Quadro 3 a seguir.

Quadro 3 - Apresentação dos artigos incluídos no âmbito do intercâmbio internacional de enfermeiros $(\mathrm{n}=4)$.

\begin{tabular}{|c|l|}
\hline \multicolumn{2}{|c|}{ INTERCÂMBIO INTERNACIONAL PROFISSIONAL } \\
\hline Autor/Ano & \multicolumn{1}{c|}{ Principais resultados } \\
\hline FOSTER, 2008. & Aperfeiçoamento de habilidades de liderança entre a equipe da maternidade . \\
\hline MESQUITA, & $\begin{array}{l}\text { Aspectos teóricos, avanço pessoal e profissional, além de estreitar laços com pesquisadores e } \\
\text { instituições do exterior para o desenvolvimento de pesquisas no seguimento da formação } \\
\text { profissional. } \\
\text { MALISKI, 2009. }\end{array}$ \\
\hline $\begin{array}{c}\text { WIEGERINK-ROE, } \\
\text { RUCKER-SHANNON, } \\
\text { 2008. }\end{array}$ & $\begin{array}{l}\text { Embora não seja realista para todos os enfermeiros, a imersão em uma outra cultura permite aos } \\
\text { mesmotir criticamente suas opiniões sobre outras culturas e sobre eles próprios. }\end{array}$ \\
\hline WRIGHT, CLOONAN, & $\begin{array}{l}\text { As enfermeiras relataram ser mais confiantes em sua capacidade de trabalhar com seus novos } \\
\text { colegas para levar novas idéias adiante com mais eficácia. }\end{array}$ \\
\hline
\end{tabular}

Fonte: Autores (2020).

\section{Discussão}

Durante a análise dos referenciais teóricos envolvidos neste estudo, percebeu-se em todos os âmbitos de formação, tanto acadêmico como profissional, que a mobilidade desenvolve no participante não apenas o saber técnico científico, mas também um forte desenvolvimento pessoal. Os estudantes e profissionais enfermeiros que vivenciaram essa experiência relataram que ao voltar para suas casas, não retornaram as mesmas pessoas que eram antes do intercâmbio, pois o fato de vivenciar outra cultura, enfrentando obstáculos, como a diferença linguística e distanciamento físico dos familiares e amigos, por exemplo, fez com que os mesmos obtivessem um maior crescimento pessoal, aprendendo a lidar com as diversas situações cotidianas. 


\subsection{Intercâmbio acadêmico internacional}

Analisando os referenciais teóricos que discutem sobre a realização de intercâmbio acadêmico em Enfermagem, Ferrillo (2020) mostrou em seu estudo que as aprendizagens das práticas clínicas de enfermagem nos serviços internacionais favorecem o desenvolvimento dos valores da profissão, tendo em vista um grande aprendizado da clínica em seu contexto geral.

Concordando com essa linha de pensamento, Couto e Pereira (2018) afirmam que a experiência da mobilidade internacional estimula nos estudantes de enfermagem o amadurecimento da profissão, refletindo assim sobre a atuação prática dos mesmos e os conhecimentos adquiridos, despertando o interesse na busca de saberes, melhorando a assistência de enfermagem dentro da heterogeneidade.

Myhre (2011) afirma que o estudante de enfermagem que realiza a prática clínica em outro país agrega mais valores à sua profissão, comparando com as práticas que são realizadas na própria cultura. Em seu estudo com os graduandos de enfermagem que participaram de intercâmbio acadêmico foi relatado pelos mesmos que houve um aumento da autoconfiança e compreensão dos conceitos fundamentais em enfermagem. Além disso, relataram também que a diferença dos idiomas não é considerada como problema, mas sim como uma forma de desenvolver ainda mais suas competências, tanto profissionais quanto pessoais. Poder comparar os diferentes sistemas de saúde envolvidos nos países é essencial para desenvolver as competências do enfermeiro.

Os autores Miles (2019), Bubadué (2013), Maas (2011), Green e colaboradores (2018), Miller et al (2010), Stone e Junlape (2014), Kaddoura, Puri e Dominick (2014), Gosse e Katic (2020), Colleen, Alistair e Susanne (1997) concordam do mesmo pensamento sobre a contribuição do intercâmbio internacional para a Enfermagem, onde afirmam que as percepções que os participantes adquirem vivenciando outros sistemas de saúde ajudam no seu crescimento profissional, desenvolvendo suas habilidades para saber solucionar problemas, tendo em vista a melhor forma possível de atender seus pacientes..

Os resultados obtidos de um estudo qualitativo descritivo realizado por Thompson, Boore e Deeny (2000) com estudantes de enfermagem que participaram de programa de mobilidade acadêmica durante a graduação mostraram que vivenciar tal experiência foi enriquecedora para os graduandos, atualmente enfermeiros, mesmo que tenham ocorrido em curto prazo, sendo suficiente para facilitar o desenvolvimento profissional e pessoal dos mesmos. Os alunos relataram ainda que foi possível melhorar o entendimento sobre os fatores culturais, saúde e os cuidados que devem ser tomados com os pacientes.

\subsection{Pós-graduação internacional em enfermagem}

No âmbito da pós-graduação que incluem mestrado e doutorado, segundo Lorenzini e colaboradores (2016), Gargia (2014), Figueredo, Fernandes e Ayala (2019), a aquisição de conhecimentos científicos ampliados, a formação de vínculos acadêmicos bem como participação em cursos, palestras e eventos no exterior favorece o desenvolvimento de habilidades interpessoais e profissionais a partir da troca de conhecimento. Experiência clínica internacional obrigatória é uma ferramenta benéfica para promover uma compreensão global do desenvolvimento acadêmico e profissional.

Embora existam programas de bolsas para pós-graduação no exterior, o número de estudantes já graduados que procuram uma especialização é menor que os graduandos em enfermagem. Decorrente disso, os autores Andrews (1988), Carty e outros autores (2009) compartilham da ideia em que no geral, os programas de educação em enfermagem não fornecem enfermeiras com a preparação acadêmica necessária para funcionar na arena internacional, logo, o principal ponto de vista defendido por eles é que o papel de liderança internacional é escasso, sendo assim necessitando de um maior apoio e preparação de enfermeiros especialistas com essa visão.

A especialização internacional é uma oportunidade para expandir perspectivas e abrir a mente para o mundo da ciência, segundo Bardaquim e Dias (2019). Conhecer outras culturas e línguas, atravessar as fronteiras e relacionar-se com 
renomados professores internacionais criando uma rede profissional de contatos internacionais contribuem positivamente na especialização do enfermeiro, atuando em resoluções de problemas e desenvolvendo essa característica fundamental de liderança em equipe.

\subsection{Intercâmbio profissional}

No aspecto de intercâmbio profissional, os estudos de Wiegerink (2008), Wright e colaboradores (2005), Foster (2009) mostram que embora não seja uma realidade comum a todos os enfermeiros, a experiência profissional em outro país despertou um olhar mais maduro desses profissionais em relação ao sistema de saúde internacional, onde o senso crítico é uma habilidade a ser aprimorada, colocando em prática a autoconfiança e papel de liderança em criação de planos estratégicos desenvolvidos com coletivamente com os demais profissionais.

\section{Considerações Finais}

A partir dos artigos estudados nesta pesquisa conclui-se que a internacionalização em enfermagem é um diferencial potencial para o enfermeiro, seja este ainda em seu processo de formação, como graduando, seja como pós-graduando, ambos tendo a oportunidade de estudar e vivenciar outras metodologias de ensino, e até mesmo de conhecer e realizar práticas de enfermagem em um país distinto do de origem.

Ademais, foi possível perceber que o maior número de participantes nos programas de mobilidade acadêmica está presente nos graduandos de enfermagem, ao comparar com o quantitativo de participantes no quesito pós graduação e profissionais enfermeiros, ou seja, costuma-se ser realizado a troca de experiências internacionais quando estes ainda estão em seu processo de formação. Apesar de ter sido a menor categorização deste estudo, é conclusivo a contribuição proveniente do intercâmbio internacional para os profissionais, no qual os enfermeiros ao exercerem sua profissão em outra cultura, trabalhando com uma equipe multiprofissional estrangeira, têm a oportunidade de comparar as diferenciar as habilidades clínicas envolvidas, podendo desse modo analisar o que pode ser melhorado, agregando mais eficiência a suas práticas profissionais.

Espera-se que com a discussão deste estudo desperte-se um maior interesse nos profissionais ou futuros profissionais de enfermagem em participar dos programas de intercâmbio, estimulando nos mesmos o desejo de enriquecer seu currículo, não se conformando apenas com as práticas curriculares que lhes foram dadas, podendo vivenciar outras aprendizagens, tanto teóricas quanto práticas, conhecendo assim diferentes sistemas de saúde e formas de exercer a prática clínica, fortalecendo seu pensamento crítico e raciocínio clínico, podendo desse modo exercer melhor suas competências como profissional enfermeiro, em suas atividades de gerenciamento e de assistência em enfermagem.

\section{Referências}

Andrews, M. M. (1988). Educational preparation for international nursing. Journal of Professional Nursing, 4(6), 430-435. https://doi.org/10.1016/S87557223(88)80094-2

Archanjo, R. (2015). Globalização e Multilingualismo no Brasil Competência Linguística e o Programa Ciência Sem Fronteiras. Revista Brasileira de Linguística Aplicada, 15(3), 621-656. http://dx.doi.org/10.1590/1984-639820156309

Barbosa, M. L. D. O., \& Neves, C. E. B. (2020). Internacionalização da educação superior: instituições e diplomacia do conhecimento. Sociologias, 22(54), 22-44. http://dx.doi.org/10.1590/15174522-104425

Bardaquim, V. A., \& Dias, E. G. (2019). A realização de intercâmbio no doutorado em enfermagem: um relato de experiência. Journal of Nursing and Health, 9(1). https://doi.org/10.15210/jonah.v9i1.14396

Caldwell, P., \& Purtzer, M. A. (2015). Long-term learning in a short-term study abroad program: "Are we really truly helping the community?". Public Health Nursing, 32(5), 577-583. https://doi.org/10.1111/phn.12168 
Carty, R., O'Grady, E. T., Wichaikhum, O. A., \& Bull, J. (2002). Opportunities in preparing global leaders in nursing. Journal of Professional Nursing, 18(2), 70-77. https://doi.org/10.1053/jpnu.2002.123725

Carvalho, J. L., Backes, D. S., Lomba, M. D. L. L. D. F., \& Colomé, J. S. (2016). Intercâmbio académico internacional: uma oportunidade para a formação do futuro enfermeiro. Revista de Enfermagem Referência, (10), 59-67. http://dx.doi.org/10.12707/RIV16018

Couto, D. S., \& Pereira, Q. L. C. (2018). Movilidad internacional en enfermería: oportunidad de ampliar la visión del mundo y agregar conocimientos. Journal of Nursing and Health, 8(2). https://doi.org/10.15210/jonah.v8i2.13623

Clark Callister, L., \& Harmer Cox, A. (2006). Opening our hearts and minds: The meaning of international clinical nursing electives in the personal and professional lives of nurses. Nursing \& Health Sciences, 8(2), 95-102. https://doi.org/10.1111/j.1442-2018.2006.00259.x

Dedee, L. S., \& Stewart, S. (2003). The effect of student participation in international study. Journal of Professional Nursing, 19(4), 237-242. https://doi.org/10.1016/S8755-7223(03)00086-3

Duffy, M. E., Farmer, S., Ravert, P., \& Huittinen, L. (2005). International community health networking project: two-year follow-up of graduates. International nursing review, 52(1), 24-31. https://doi.org/10.1111/j.1466-7657.2004.00252.x

Dyches, C., Haynes-Ferere, A., \& Haynes, T. (2019). Fostering cultural competence in nursing students through international service immersion experiences. Journal of Christian Nursing, 36(2), E29-E35. doi: 10.1097 / CNJ.0000000000000602

Fennell, R. (2009). The impact of an international health study abroad program on university students from the United States. Global health promotion, 16(3), 17-23. https://doi.org/10.1177/1757975909339766

Ferrillo, H. (2020). Measuring professional nursing value development in students participating in international service learning: A quasi-experimental study. Nurse education today, 84, 104221. https://doi.org/10.1016/j.nedt.2019.104221

Figueredo, L. P., Fernandes, M. D. F. D. P., \& Ayala, O. A. (2019). Intercâmbio Acadêmico Internacional na Pós-Graduação em Enfermagem: Relato de Experiência. Revista de Divulgação Científica Sena Aires, 8(4), 512-517.

Foster, J. (2009). Cultural humility and the importance of long-term relationships in international partnerships. Journal of Obstetric, Gynecologic \& Neonatal Nursing, 38(1), 100-107. https://doi.org/10.1111/j.1552-6909.2008.00313.x

Girondi, J. B. R., Cerqueira, M. L., Amante, L. N., Hammerschmidt, K. S. D. A., Fernandez, D. L. R., \& Palma, A. P. L. B. Z. (2016). Intercâmbio acadêmico internacional em enfermagem perioperatória: relato de experiência na graduação. Revista Mineira de Enfermagem, 20. DOI: http://www.dx.doi.org/10.5935/1415-2762.20160013

Gosse, N. L., \& Katic-Duffy, A. (2020). Nursing student and faculty perceptions of reciprocity during international clinical learning experiences: A qualitative descriptive study. Nurse education today, 84, 104242. https://doi.org/10.1016/j.nedt.2019.104242

GUSKUMA, Erica Mayumi et al. Mobilidade acadêmica internacional na formação em enfermagem: relato de experiência. Revista Brasileira de Enfermagem, v. 69, n. 5, p. 986-990, 2016. http://dx.doi.org/10.1590/0034-7167-2015-0128

Green, B. F., Johansson, I., Rosser, M., Tengnah, C., \& Segrott, J. (2008). Studying abroad: A multiple case study of nursing students' international experiences. Nurse Education Today, 28(8), 981-992. https://doi.org/10.1016/j.nedt.2008.06.003

Hinshaw, A. S. (1997). International facilitation of doctoral education in nursing. Journal of professional nursing: official journal of the American Association of Colleges of Nursing, 13(4), 209. https://pubmed.ncbi.nlm.nih.gov/9239976/.

Holtbrügge, D., \& Engelhard, F. (2016). Study abroad programs: Individual motivations, cultural intelligence, and the mediating role of cultural boundary spanning. Academy of Management Learning \& Education, 15(3), 435-455. https://doi.org/10.5465/amle.2015.0128

Kaddoura, M., Puri, A., \& Dominick, C. A. (2013). International academic service learning: Lessons learned from students' travel experiences of diverse cultural and health care practices in Morocco. Journal of Nursing Education, 53(1), 45-47. https://doi.org/10.3928/01484834-20131223-01

Jung, B. C., Freitag, V. L., Gonzáles, R. I. C., \& Dalmolin, I. S. (2015). Colômbia: cenário de estudo em enfermagem no programa de mobilidade acadêmica internacional. Revista de Enfermagem da UFSM, 5(4), 675-682. http://dx.doi.org/10.5902/2179769218952

Kako, P. M., \& Klingbeil, C. G. (2019). Facilitating Cultural Humility and Attunement for Nursing and Health Professions Students Through a Study Abroad Program in Kenya. Nursing education perspectives, 40(5), 278-282. doi: 10.1097 / $01 . N E P .0000000000000561$

Kohlbry, P. W. (2016). The impact of international service-learning on nursing students' cultural competency. Journal of Nursing Scholarship, 48(3), 303-311. https://doi.org/10.1111/jnu.12209

Law, K., \& Muir, N. (2006). The internationalisation of the nursing curriculum. Nurse Education in Practice, 6(3), 149-155. https://doi.org/10.1016/j.nepr.2005.11.004

Koskinen, L., \& Jokinen, P. (2007). Multicultural healthcare: A transatlantic project. Nurse Educator, 32(2), 89-93. doi: 10.1097 / 01.NNE.0000264330.63662.cd

Lee, N. J. (2004). The impact of international experience on student nurses' personal and professional development. International Nursing Review, 51(2), 113122.

https://doi.org/10.1111/j.1466-7657.2003.00200.x

Lorenzini, E., Oelke, N. D., Marck, P. B., \& Dall'Agnol, C. M. (2016). Contribuições do programa de doutorado sanduíche nas abordagens metodológicas: relato de experiência. Revista Gaúcha de Enfermagem, 37(2). https://doi.org/10.1590/1983-1447.2016.02.58244 
Maas, L. G. (2011). Benchmarking one's health care system: Professional development through an international experience. Nurse Education in Practice, 11(5), 293-297. https://doi.org/10.1016/j.nepr.2011.01.005

Maas, L. G., \& Ezeobele, I. E. (2014). Reflections from Dutch advanced nursing practice students on psychiatric mental healthcare in the United States. Archives of Psychiatric Nursing, 28(6), 357-361. https://doi.org/10.1016/j.apnu.2014.08.008

Miles, L. W., Erämaa, S., \& Parkkinen, S. (2019). "The World Is Our Campus”: Student Satisfaction with an International Nursing Education Partnership. Nursing education perspectives, 40(5), 300-302. doi: 10.1097 / 01.NEP.00000000000000567

Morgan, D. A. (2019). Learning in liminality. Student experiences of learning during a nursing study abroad journey: A hermeneutic phenomenological research study. Nurse education today, 79, 204-209. https://doi.org/10.1016/j.nedt.2019.05.036

Mwenda, A. S. (2012). A Student's Analysis of the Moi University-Linköping University Exchange Programme. Education for Health, $25(2), 111$. https://www.educationforhealth.net/text.asp?2012/25/2/111/103458

Myhre, K. (2011). Exchange students crossing language boundaries in clinical nursing practice. International nursing review, 58(4), 428-433. https://doi.org/10.1111/j.1466-7657.2011.00904.x

Nora, C. R. D., Schaefer, R., Schveitzer, M. C., Zoboli, E. L. C. P., \& Vieira, M. M. (2018). Dupla titulação em enfermagem: potencialidades e desafios de uma experiência de internacionalização acadêmica discente. Revista da Escola de Enfermagem da USP, 52. https://doi.org/10.1590/s1980$220 x 2017019803311$

Ortega, J., Mitchell, EM, \& Peragallo, N. (2016). Além das fronteiras: educação global em enfermagem para o futuro. Perspectivas de educação em enfermagem, 37 (4), 227-229. doi: 10.5480 / 13-1296

Pinto, M. M., \& Larrechea, E. M. (2018). Internacionalização da educação superior: uma análise das tendências de mobilidade dos estudantes entre países do norte e do sul global. Avaliação: Revista da Avaliação da Educação Superior (Campinas), 23(3), 718-735. http://dx.doi.org/10.1590/s141440772018000300009

Rolls, C., Inglis, A., \& Kristy, S. (1997). Study abroad programs: creating awareness of and changing attitudes to nursing, health and ways of living in other cultures. Contemporary Nurse, 6(3-4), 152-156. https://doi.org/10.5172/conu.1997.6.3-4.152

Ryan, M., \& Twibell, R. S. (2002). Outcomes of a transcultural nursing immersion experience: Confirmation of a dimensional matrix. Journal of Transcultural Nursing, 13(1), 30-39. https://doi.org/10.1177/104365960201300106

Rosa Mesquita, M. G., Moreira, M. C., \& Maliski, S. (2009). Em busca de conhecimento de enfermagem sobre o homem com câncer: uma experiência internacional. Escola Anna Nery Revista de Enfermagem, 13(2), 421-424.

da Silva Cavalcante, I. M., Guedes, G. F., \& de Araújo Püschel, V. A. (2018). Mobilidade internacional estudantil de Enfermagem da Universidade de São Paulo. Revista Brasileira de Enfermagem, 71.

Smith, K., \& Curry, K. (2011). Is it worth it? Measuring the long-term effects of an international experience for nursing students in Ecuador. Journal of Community Health Nursing, 28(1), 14-22.

Smith-Miller, C. A., Leak, A., Harlan, C. A., Dieckmann, J., \& Sherwood, G. (2010, January). "Leaving the comfort of the familiar": Fostering workplace cultural awareness through short-term global experiences. In Nursing Forum (Vol. 45, No. 1, pp. 18-28). Malden, USA: Blackwell Publishing Inc. https://doi.org/10.1111/j.1744-6198.2009.00163.x

Steen, S., \& Zdechlik, L. (2016). A nursing semester in a developing country: lessons learned. Nurse educator, 41(5), 266-269. doi: 10.1097 / NNE.0000000000000263

Stone, T. E., Francis, L., Van der Riet, P., Dedkhard, S., Junlapeeya, P., \& Orwat, E. (2014). Awakening to the other: Reflections on developing intercultural competence through an undergraduate study tour. Nursing \& health sciences, 16(4), 521-527.

https://doi.org/10.1111/nhs.12139

Thompson, K., Boore, J., \& Deeny, P. (2000). A comparison of an international experience for nursing students in developed and developing countries. International Journal of Nursing Studies, 37(6), 481-492. https://doi.org/10.1016/S0020-7489(00)00027-4

Ulvund, I., \& Mordal, E. (2017). The impact of short term clinical placement in a developing country on nursing students: A qualitative descriptive study. Nurse Education Today, 55, 96-100. https://doi.org/10.1016/j.nedt.2017.05.013

UNESCO, United Nations Educational, Scientific and Cultural Organization Institute for Statistics. (n.d.). UIS statistics. http://data.uis.unesco.org/.

Westerbotn, M., Kneck, Å., Hovland, OJ, Elrond, M., Pedersen, I., Lejonqvist, GB, ... \& Sigurdardottir, Á. K. (2015). Participar da colaboração nórdica; Experiências e percepções dos estudantes de enfermagem na perspectiva da aprendizagem: um estudo qualitativo. Nurse Education Today , 35 (5), 712-717. https://doi.org/10.1016/j.nedt.2015.01.025

Wiegerink-Roe, E., \& Rucker-Shannon, M. (2008). Immersion in China: lessons learned. Nurse educator, 33(2), 71-74. doi: 10.1097 / 01.NNE.0000299510.93517.b8

Wright, S., Cloonan, P., Leonhardy, K., \& Wright, G. (2005). An international programme in nursing and midwifery: building capacity for the new millennium. International Nursing Review, 52(1), 18-23. https://doi.org/10.1111/j.1466-7657.2004.00250.x

Ximenes, N. F. R. G., Lopes, N. D., Cunha, I. C. K. O., Ribeiro, M. A., Freire, N. P., Kalinowski, C. E., ... \& Albuquerque, I. M. A. N. (2019). Reflexões sobre a formação em Enfermagem no Brasil a partir da regulamentação do Sistema Único de Saúde. Ciência \& Saúde Coletiva, 25, 37-46. https://doi.org/10.1590/1413-81232020251.27702019 